Artigo Original

\title{
Impacto do treinamento resistido na força e hipertrofia muscular em HIV-soropositivos
}

\author{
Ciro José Brito ${ }^{1}$ \\ Edmar Lacerda Mendes ${ }^{2}$ \\ Aparecido Pimentel Ferreira ${ }^{4}$ \\ Sérgio Oliveira De Paula ${ }^{3}$ \\ Otávio de Tolêdo Nóbrega ${ }^{5}$ \\ Cláudio Córdova ${ }^{6}$ \\ ${ }^{1}$ Departamento de Educação Física, Centro de Ciências Biológicas e da Saúde, Universidade \\ Federal de Sergipe, Aracaju, SE, Brasil \\ ${ }^{2}$ Departamento de Ciências do Esporte, Universidade Federal do Triângulo Mineiro, Uberaba, MG, \\ Brasil \\ ${ }^{3}$ Departamento de Biologia Geral, Centro de Ciências Biológicas e da Saúde, Universidade Federal \\ de Viçosa, Vicosa, MG, Brasil \\ ${ }^{4}$ Departamento de Educação Física, Universidade Paulista; Núcleo Interdisciplinar de Pesquisa - \\ NIP das Faculdades PROMOVE de Brasília, DF, Brasil \\ ${ }^{5}$ Faculdade de Ceilândia, Universidade de Brasília, Brasília, DF, Brasil \\ ${ }^{6}$ Pró-Reitoria de Pós-Graduação e Pesquisa, Universidade Católica de Brasília, Brasilia, DF, Brasil
}

\begin{abstract}
Resumo: O presente estudo investigou o efeito de 24 semanas de treinamento resistido (TR) sobre a força e hipertrofia muscular de pacientes HIV-soropositivos. Participaram deste estudo 45 voluntários submetidos à terapia antirretroviral fortemente ativa (HAART), destes, 23 realizaram 3 sessões semanais, com 10 repetições a $80 \% 1 \mathrm{RM}$. O teste de 1RM foi realizado de acordo com a metodologia proposta por Kraemer e Fry (1995), para estimativa da hipertrofia muscular adotou-se as equações de Frisancho (1984). Em comparação aos valores, o TR melhorou a força de $1 \mathrm{RM}$ nos exercícios de agachamento em $49 \%(21,0 \pm 4,9$ vs. $31,2 \pm 5,1 ; P=0,001)$, supino reto em $13 \%$ $(34,3 \pm 8,1$ vs. $39,8 \pm 9,4 ; P=0,04)$, cadeira extensora em $34,1 \%(26,3 \pm 7,1$ vs. $37,1 \pm 6,6 ; P=0,01)$, tríceps em $51 \%$ $(22,9 \pm 4,0$ vs. $38,3 \pm 4,9 ; P=0,001)$, pulley costas em $31,5 \%(31,7 \pm 3,9$ vs. $41,7 \pm 4,4 ; P=0,01)$, cadeira flexora em $37,2 \%(18,9 \pm 3,4$ vs. $27,3 \pm 3,2 ; P=0,01)$ e rosca bíceps em $60 \%$ (27,9 $9 \pm 6,9$ vs. $40,4 \pm 4,5 ; P=0,001)$. Não foram observadas diferenças $(P<0,05)$ entre os valores basais e finais para o grupo controle. Observou-se aumento significativo $(P<0,05)$ na área muscular do braço isenta de massa óssea, no grupo TR $\left(52,8 \pm 14,5 \mathrm{~cm}^{2}\right)$ em relação ao controle $\left(39,5 \pm 12,4 \mathrm{~cm}^{2}\right)$. Ademais, o TR resultou em significativa $(P<0,05)$ redução da glicemia sanguínea de jejum $(96,5 \pm 18,3$ vs. 90,5 $\pm 12,6)$, pressão arterial sistólica $(126,3 \pm 14,3$ vs. 120,0 $\pm 10,0)$ e circunferência de cintura $(83,0 \pm 12,5$ vs. $80,6 \pm 10,2)$. Conclui-se que seis meses de TR resultaram em melhora na força e hipertrofia, ademais, o treinamento aplicado contribuiu para a regulação das variáveis metabólicas dos pacientes. Uma vez que a HAART é inevitável ao HIV-soropositivo, recomenda-se que o exercício físico seja realizado no intuito de dirimir os efeitos colaterais advindos desta terapia.
\end{abstract}

Palavras-chave: Terapia Anti-Retroviral de Alta Atividade. Síndrome de lipodistrofia associada ao HIV. Terapia por exercício. Antropometria.

\section{Impact of resistance training on strength and muscle hypertrophy in HIV-seropositive}

\begin{abstract}
The aim of this study was to investigate the effect of 24 weeks of resistance training (RT) on the strength and muscle hypertrophy in patients with HIV-seropositive. Participated in the study 45 subjects undergoing highly active antiretroviral therapy (HAART). They were divided into two groups: control $(n=22)$ and RT $(n=23)$. The RT group realized three sessions, with 10 repetitions at $80 \%$ of $1 \mathrm{MR}$. The MR tests were performed pre and post 24 weeks according to Kraemer and Fry (1995) and the equations proposed by Frisancho (1984) were adopted to estimate bone-free upper arm muscle area. Compared to baseline, the RT improved the strength of $1 \mathrm{MR}$ in the squat exercise at $49 \%(21.0 \pm 4.9$ vs. $31.2 \pm 5.1 ; P=0.001)$, bench press by $13 \%(34.3 \pm 8.1$ vs. $39.8 \pm 9.4, P=0.04)$, leg extension in $34.1 \%(26.3 \pm 7.1$ vs. $37.1 \pm 6.6, P=0.01)$, triceps in $51 \%(22.9 \pm 4.0$ vs. $38.3 \pm 4.9, P=0.001)$, pulley in $31.5 \%$ $(31.7 \pm 3.9$ vs. $41.7 \pm 4.4, P=0.01)$, leg curl in $37.2 \%(18.9 \pm 3.4$ vs. $27.3 \pm 3.2, P=0.01)$ and biceps in $60 \%(27.9 \pm 6.9$ vs. 40.4 $\pm 4.5, P=0.001)$; there were no significant differences between baseline and final at control. The Bone-free upper arm muscle area at RT group $\left(52.8 \pm 14.5 \mathrm{~cm}^{2}\right)$ was significant increased $(P<0.05)$ comparing to control $(39.5 \pm 12.4$ $\left.\mathrm{cm}^{2}\right)$. Moreover, the RT resulted in significant reduction $(P<0.05)$ in fasting blood glucose $(96.5 \pm 18.3 \mathrm{vs}$. 90.5 \pm 12.6 , $\mathrm{P}<0.05)$, systolic blood pressure $(126.3 \pm 14.3 \mathrm{mmHg}$ vs. $120.0 \pm 10.0 \mathrm{mmHg})$ and waist circumference $(83.0 \pm 12.5 \mathrm{~cm}$ vs. $80.6 \pm 10.2 \mathrm{~cm})$. We conclude that six months of RT resulted in improvement in strength and hypertrophy; in addition, this training contributed to regulate the metabolic variables from these patients. Since the HAART is inevitable to HIV-seropositive, It's recommended that physical exercise be realized to minimize the side effects from this therapy.
\end{abstract}

Keywords: High Activity Anti-Retroviral Therapy. HIV-lipodystrophy Syndrome. Exercise Therapy. Anthropometry. 


\section{Introdução}

O vírus da imunodeficiência humana (HIV) está presente em todos os continentes, com maior prevalência de infecção na África subsaariana, onde alcança status de pandemia. De acordo com os dados apresentados pela Organização Mundial da Saúde, há um milhão e meio de soropositivos na América Latina, dentre os quais seiscentos mil são brasileiros. No ano de 2007, ocorreram cerca de cem mil infecções e cinquenta e oito mil mortes na América Latina associadas à infecção pelo HIV (UNAIDS, 2008).

A terapia antirretroviral fortemente ativa (HAART) tem proporcionado melhora na qualidade de vida e aumento na sobrevida aos soropositivos mediante supressão do HIV (COUZIGOU et al., 2007). Entretanto, tem sido reportado que soropositivos submetidos a esta terapia podem desenvolver síndrome lipodistrófica por infecção pelo HIV (DELL'ISOLA et al., 2006; DUONG et al., 2006). Esta síndrome é reconhecida como um dos efeitos adversos associado aos inibidores de protease em terapia de longa duração (DUONG et al., 2006). Outra droga associada ao tratamento, definida como inibidores de transcriptase reversa, relaciona-se também às alterações lipodistrotóficas do tipo lipoatrofia (DELL'ISOLA et al., 2006) e toxicidade mitocondrial, contribuindo negativamente para a elevação da acidose lática (KOHLER; LEWIS, 2007).

Adicionalmente, outra indesejável alteração, decorrente da infecção pelo HIV, consiste na progressiva redução da massa corporal magra (MACERA, 2007) que, por sua vez, associa-se à redução da capacidade do sistema músculoesquelético em gerar força (O'BRIEN et al., 2004). Uma vez que a HAART per se não resulta em hipertrofia muscular (CADE et al., 2007), novos estudos devem ser realizados com o propósito de elucidar acuradas prescrições de exercícios resistidos, visando a efeitos positivos do treinamento na constituição de massa muscular magra e ganho de força em pacientes soropositivos (SATTLER et al., 2002; $\underline{\text { SOUZA et }}$ al., 2008). Ademais, revisão de literatura tem sugerido limitações como ausência de grupocontrole (ROBINSON et al., 2007; SOUZA et al., 2008), amostras pequenas (RØGE et al., 2002; ROBINSON et al., 2007; SOUZA et al., 2008) e reduzido período de intervenção (LINDEGAARD et al., 2008; SAKKAS et al., 2009).
O TR, por sua vez, pode ser usado para evitar a perda da massa magra e a perda funcional do músculo, uma vez que um dos efeitos crônicos clássicos do TR, importante para esta população, é o aumento da força (MENDES et al., 2011), oriundo tanto pelo aumento da área transversa muscular (DOLAN et al., 2006), quanto pela melhora da eficiência muscular (DOLAN et al., 2006; MENDES et al., 2011; SOUZA et al., 2011). Diante do exposto, o presente estudo tem por objetivo investigar o efeito de 24 semanas de TR em medidas antropométricas, força e hipertrofia muscular de pacientes soropositivos com avaliação dos hábitos alimentares e a prescrição dietética realizada por nutricionista.

\section{Materiais e Métodos}

\section{Casuística}

Trata-se de uma amostra de indivíduos HIV/AIDS submetidos à HAART, constituída por conveniência. Para o presente estudo foi incluído, inicialmente, infectados, há pelo menos 1 ano $(n=150)$, independente da presença de lipodistrofia. Destes, 64 não desejaram participar do estudo. Ademais, foram excluídos aqueles que não completaram as etapas previstas ou abandonaram 0 estudo $(n=4)$; apresentaram antecedentes de hipertensão $(n=4)$; portavam doenças cardíacas ou diabetes $(n=2)$;eram fumantes, consumidores de bebidas alcoólicas ou de fármacos de ação diurética $(n=23)$; estavam sob tratamento com corticóides, esteróides anabolizantes ou hormônio do crescimento $(n=2)$; apresentavam doenças oportunistas (infecções ou tumores) nos três meses que antecederam o experimento $(n=5)$ ou realizaram alguma cirurgia como liposucção ou lipoescultura seis meses antes do experimento $(n=1)$. Finalmente, após a triagem inicial e aplicação dos critérios de exclusão, a amostra foi constituída por 45 voluntários. As características sócio-demográficas e epidemiológicas da amostra foram obtidas por meio da versão abreviada do Questionário de Qualidade de Vida da Organização Mundial de Saúde para soropositivos, traduzido e validado para a língua portuguesa (ZIMPEL; FLECK, 2007).

O estudo foi previamente aprovado pelo comitê de ética para pesquisas em seres humanos, da Universidade Federal de Viçosa MG, Brasil, de acordo com a declaração de Helsinki. Os voluntários foram informados sobre os objetivos do estudo e assinaram um termo de 
consentimento livre e esclarecido. Todos os voluntários mantiveram a HAART seguindo recomendações médicas e foram monitorados por médicos, nutricionista e profissional de educação física durante a investigação. Para determinação de presença de lipodistrofia, adotou-se a metodologia descrita por Lichtenstein et al. (2001). Posteriormente, os pacientes foram randomicamente alocados para os grupos TR ou controle, mediante utilização do software GraphPad StatMate, versão 1.01, San Diego USA. Após o sorteio, um dos pesquisadores (cego aos objetivos do estudo) entrou em contato com os participantes, por telefone, a fim de orientá-los quanto às atividades e o cronograma a serem desenvolvidos (TR ou Controle). É importante salientar que o sorteio, mediante programas computadorizados baseados em algoritmos pseudo-randomizados, exclui possíveis vieses de seleção por parte dos pesquisadores.

\section{Intervenção}

Trata-se de um delineamento quasiexperimental com pré e pós-testes. A intervenção consistiu em 24 semanas de TR, precedida de uma semana de adaptação. Os seguintes exercícios foram selecionados e prescritos segundo orientações do American College of Sports Medicine: supino, agachamento, pulley costas, mesa flexora, rosca bíceps, tríceps e cadeira extensora (ACSM, 2009).

Utilizou-se o teste de uma repetição máxima (1RM) para a prescrição do exercício e avaliação do desempenho da força muscular pré e pósintervenção (Kraemer e Fry, 1995). Após aquecimento específico, o teste iniciou-se com carga de 40 a $60 \%$ de 1RM estimada e subsequentes, com intervalo de três minutos de descanso. Considerou-se 1RM a carga atingida na tentativa anterior à falha concêntrica. Todos os testes foram realizados por um avaliador experiente, em três dias de atividade, sendo que, no primeiro, realizaram-se os exercícios supino reto e cadeira extensora. No dia seguinte, rosca bíceps e agachamento. No ultimo dia, pulley costas, mesa flexora e tríceps.

O treinamento foi prescrito por um único pesquisador acompanhado de dois auxiliares. A fase de aquecimento foi realizada anteriormente a todas as sessões e consistiu de 5 minutos de exercício, em esteira, com variação entre os índices 11 e 12 da escala de Borg (BORG, 1982). $\mathrm{Na}$ semana de adaptação, os voluntários realizaram três sessões, onde cada continham três séries de 6 a 8 repetições, por exercício, a $70 \%$ de 1 RM com intervalos de 2 minutos entre séries. Ao longo das 24 semanas foram realizadas 3 séries de $8-10$ repetições a $80 \%$ de 1RM, com intervalos de 1 minuto entre séries, de acordo com as recomendações do American College of Sports Medicine para adultos saudáveis (ACSM, 2009). A carga de exercício foi progressivamente alterada de acordo com a evolução de cada participante (AGIN et al., 2001).

\section{Avaliação}

\section{Medidas antropométricas}

Todas as medidas foram realizadas sem a identificação do voluntário (delianeamento cego) e por um único avaliador experiente. Para evitar possíveis vieses, o avaliador desta etapa não foi o mesmo que prescreveu o treinamento. As variáveis antropométricas foram medidas antes e depois de 24 semanas de intervenção. A massa corporal foi medida com resolução de $100 \mathrm{~g}$ (Filizola, Brasil) e a estatura, pelo estadiômetro acoplado na balança, com resolução de $1 \mathrm{~cm}$. Estas variáveis foram usadas para calcular 0 índice de massa corporal. As dobras cutâneas foram mensuradas em triplicata e alternadas do lado direito do corpo, adotando-se a média das três dobras. Quando a diferença entre as medidas era superior a $5 \%$, realizou-se uma quarta medida e descartou-se o valor discrepante (PETROSKI, 2009). O percentual de gordura foi calculado por meio das equações específicas propostas por Florindo et al. (2008). Utilizaram-se as equações que apresentaram maior correlação $(r)$ e coeficiente de determinação $\left(R^{2}\right)$ e menor erro padrão de estimativa (EPE), frente ao padrãoouro (DEXA).

\section{Homens:\%G $=3,385+0,279(M A+S B)(r=0,92$, $R^{2}=0,83$ e $E P E=2,42 \%$ ) Mulheres:\%G $=-53,734+0,582$ \\ $(B I+T R+S B+M A+S I+A B+C X)\left(r=0,92, R^{2}=0,83 \mathrm{e}\right.$ $E P E=2,42 \%)$}

Onde: $\% \mathrm{G}=$ percentual de gordura, $\mathrm{BI}=$ bicipital, $\mathrm{TR}=$ triciptal, $\mathrm{SB}=$ subescapular, $\mathrm{MA}=$ medial axilar, $\mathrm{SI}=$ supra ilíaca, $\mathrm{AB}=$ abdominal, $\mathrm{CX}=$ coxa medial.

A partir do \%G e da massa corporal, estimouse a massa corporal magra e a gorda. Adicionalmente, foram medidos os perímetros de braço, pescoço, cintura e quadril de acordo com a metodologia descrita por Petroski (2009). Para 
estimativa da hipertrofia muscular do braço, adotaram-se as equações de Frisancho (1984):

$$
\begin{gathered}
\text { Homens: } A M B\left(\mathrm{~cm}^{2}\right)=\{[C B R(\mathrm{~cm})-\pi T r i \\
\left.(\mathrm{cm})]^{2} / 4 \pi\right\}-10,0 \\
\text { Mulheres: } A M B\left(\mathrm{~cm}^{2}\right)=\{[C B R(\mathrm{~cm})-\pi T r i \\
\left.(\mathrm{cm})]^{2} / 4 \pi\right\}-6,5
\end{gathered}
$$

Onde: $A M B$ - área muscular do braço isenta de massa óssea, CBR - circunferência do braço relaxado, Tri - espessura do tríceps.

\section{Medidas bioquímicas}

Amostras de sangue $(5 \mathrm{ml})$ foram coletadas na veia antecubital, armazenadas em tubos com EDTA e heparina (Becton Dickinson, São Paulo, SP, Brasil) e centrifugadas a 3.400 RPM por 10 min para separar o plasma. As variáveis bioquímicas foram constituídas por glicemia sanguínea, linfócitos $\mathrm{CD}^{+}, \mathrm{CD}^{+}$e carga viral. A glicemia foi analisada pelo método Enzimáticocolorimétrico, com kit Human GMBH e aparelho Autohumalyzer $\quad A 5^{\circledR}$ específico (Abbott laboratories, Abbott Park, IL, Estados Unidos da América). Por citometria de fluxo em metodologia multiteste, foi possível dosar a contagem de células $\mathrm{CD}^{+}{ }^{+} \mathrm{eD}^{+}$(Sistema FACSCalibur ${ }^{\circledR}$, Becton Dickinson, San Jose, CA, Estados Unidos da América). A carga viral de cada paciente foi determinada por ensaio ultra-sensível de detecção do RNA viral em sistema Amplicor HIV-1 monitor assay ${ }^{\circledR}$ (Roche Molecular Systems, Branchburg, NJ, Estados Unidos da América) com faixa de detecção entre 50 e 500.000 cópias de RNA por ml.

\section{Variáveis hemodinâmicas}

A variável frequência cardíaca de repouso foi mensurada por cardio-frequencímetro Polar $\mathrm{F}^{\circledR}$, (Polar Systems, Kempele, OU, Finlândia). A pressão arterial foi estimada em duplicata pelo método auscultatório em esfigmomanômetro aneróide (Premium, São Paulo, SP, Brasil). As médias pressóricas foram registradas segundo recomendações das $\mathrm{V}$ Diretrizes Brasileiras de Cardiologia (SBC, 2007).

\section{Controle nutricional}

Os procedimentos descritos neste tópico foram realizados por um nutricionista. Inicialmente, os hábitos alimentares foram avaliados por meio de questionário e de recordatório habitual da dieta. Posteriormente, prescreveu-se dieta individualizada com as demandas energéticas (necessidade energética estimada) calculadas mediante a ingestão de macro-nutrientes, em conformidade com as variáveis gênero, idade, massa corporal, estatura e nível de atividade física (IOM, 2005). O nível de atividade física foi estimado pelo questionário International Physical Activity Questionary (MATSUDO et al., 2001). O teor energético da dieta recomendada atendia de 90 a $110 \%$ da necessidade energética estimada.

As recomendações nutricionais implementadas consideraram ainda a adequação da ingestão de alguns micro-nutrientes tais como ferro, vitamina $C$, vitamina $A$, niacina, tiamina e riboflavina calculadas com base nas Dietary Reference Intakes (IOM, 2000; IOM, 2005), considerando-se a ingestão estimada de cada nutriente como melhor ponto de adequação (Estimated Average Requerement). Para fibras e cálcio, utilizou-se como parâmetro a ingestão adequada (Adequate Intake). Para o consumo de colesterol, adotou-se o ponto de corte preconizado pela Sociedade Brasileira de Cardiologia (SBC, 2007). As dietas foram calculadas utilizando-se o programa Diet-Pro versão 4.0 configurada para a Tabela de Composição Química de Alimentos do Instituto Brasileiro de Geografia e Estatística.

\section{Análises estatísticas}

Realizou-se análise exploratória dos dados para identificação e correção de valores extremos conforme metodologia sugerida por Tabachinick e Fidell (2001). O teste de Shapiro-Wilk foi utilizado para testar a hipótese de normalidade dos dados. Adotou-se 0 teste de analise de covariância (ANCOVA) para comparação entre as médias dos grupos, tendo como covariante medidas de préteste. Quando apropriado, o teste do Quiquadrado $\left(\chi^{2}\right)$ foi utilizado para verificar diferenças significativas nas frequências percentuais. $\mathrm{Na}$ impossibilidade deste teste $(n<5)$, adotou-se a prova exata de Fischer. Em todas as análises utilizou-se nível de significância ( $\alpha) P<0,05$.

\section{Resultados}

As características sócio-demográficas, epidemiológicas e nível prévio de atividade física são apresentadas na Tabela 1. 
Tabela 1. Distribuição de frequências para características sócio-demográficas e epidemiológicas.

\begin{tabular}{|c|c|c|c|c|c|}
\hline & \multicolumn{2}{|c|}{$\operatorname{TR}(n=23)$} & \multicolumn{2}{|c|}{ Controle $(n=22)$} & \multirow[t]{2}{*}{$P$} \\
\hline & $\mathbf{N}$ & $\%$ & $\mathbf{N}$ & $\%$ & \\
\hline \multicolumn{6}{|l|}{ Gênero } \\
\hline Masculino & 7 & 30,4 & 7 & 31,8 & 0,92 \\
\hline Feminino & 16 & 69,6 & 15 & 68,2 & \\
\hline \multicolumn{6}{|l|}{ Lipodistrofia } \\
\hline Positivo & 12 & 52,2 & 11 & 50,0 & 0,884 \\
\hline Negativo & 11 & 47,8 & 11 & 50,0 & \\
\hline \multicolumn{6}{|l|}{ Renda individual } \\
\hline Até 1 salário mínimo & 12 & 52,2 & 13 & 59,1 & 0,641 \\
\hline > 1 salário mínimo & 11 & 47,8 & 9 & 40,9 & \\
\hline \multicolumn{6}{|l|}{ Grau de instrução } \\
\hline Nenhum & 3 & 13,0 & 5 & 22,7 & 0,394 \\
\hline Alfabetizados & 20 & 87,0 & 17 & 77,3 & \\
\hline \multicolumn{6}{|l|}{ Estado civil } \\
\hline Solteiros/separados/divorciados/viúvos & 14 & 60,9 & 12 & 54,6 & 0,668 \\
\hline Casado ou vivendo como casado & 9 & 39,1 & 10 & 45,4 & \\
\hline \multicolumn{6}{|l|}{ Tempo conhecido de infecção } \\
\hline$<5$ anos & 9 & 39,1 & 6 & 27,3 & 0,399 \\
\hline$>5$ anos & 14 & 60,9 & 16 & 72,7 & \\
\hline \multicolumn{6}{|l|}{ Nível de atividade física } \\
\hline Irregularmente ativo & 1 & 4,3 & 1 & 4,5 & 0,769 \\
\hline Sedentário & 22 & 95,7 & 21 & 95,5 & \\
\hline
\end{tabular}

Dados apresentados em valores absolutos e porcentagem.

Tabela 2. Medidas antropométricas, composição corporal, metabólicas, imunitárias e ingestão alimentar para os grupos TR e Controle.

\begin{tabular}{|c|c|c|c|c|}
\hline & TR $(n$ & $=23)$ & Controle & $(n=22)$ \\
\hline & Pré & Pós & Pré & Pós \\
\hline Antropometria & & & & \\
\hline Massa Corporal, kg & $69,1 \pm 14,0$ & $64,9 \pm 11,3^{*}$ & $70,1 \pm 13,6$ & $72,7 \pm 8,2$ \\
\hline Estatura, m & $1,7 \pm 0,1$ & --- & $1,6 \pm 0,1$ & --- \\
\hline $\mathrm{IMC}, \mathrm{Kg} / \mathrm{m}^{2}$ & $25,3 \pm 4,3$ & $23,8 \pm 3,6 \pi$ & $26,5 \pm 6,2$ & $27,5 \pm 4,4$ \\
\hline$\%$ de Gordura & $25,2 \pm 5,9$ & $19,8 \pm 4,1^{\star}$ & $27,2 \pm 6,8$ & $28,5 \pm 5,0$ \\
\hline MG, kg & $17,4 \pm 7,3$ & $12,9 \pm 4,0 \pi$ & $19,1 \pm 7,0$ & $20,7 \pm 4,8$ \\
\hline $\mathrm{MM}, \mathrm{kg}$ & $51,7 \pm 9,5$ & $52,0 \pm 8,0$ & $51,0 \pm 8,9$ & $52,0 \pm 5,9$ \\
\hline Perímetro de braço, $\mathrm{cm}$ & $30,4 \pm 4,3$ & $31,5 \pm 3,6$ & $30,3 \pm 4,4$ & $29,4 \pm 3,4$ \\
\hline Perímetro de pescoço, $\mathrm{cm}$ & $35,4 \pm 3,2$ & $34,2 \pm 2,0$ & $35,2 \pm 3,1$ & $35,2 \pm 2,6$ \\
\hline Perímetro de Cintura, $\mathrm{cm}$ & $83,0 \pm 12,5$ & $80,6 \pm 10,2^{*}$ & $86,2 \pm 13,6^{* *}$ & $88,1 \pm 11,7^{n *}$ \\
\hline Perímetro de Quadril, cm & $98,2 \pm 11,4$ & $96,4 \pm 9,5$ & $97,9 \pm 12,5$ & $99,3 \pm 9,7$ \\
\hline Pressão arterial e frequên & & & & \\
\hline $\mathrm{PAS}, \mathrm{mmHg}$ & $126,3 \pm 14,3$ & $120,0 \pm 10,0^{*}$ & $123,9 \pm 12,3$ & $126,6 \pm 9,7$ \\
\hline $\mathrm{PAD}, \mathrm{mmHg}$ & $81,1 \pm 9,5$ & $78,3 \pm 7,8$ & $82,0 \pm 9,3$ & $80,2 \pm 9,6$ \\
\hline FCR, bpm & $76,8 \pm 8$ & $75,4 \pm 7,2$ & $76,5 \pm 11,1$ & $79,8 \pm 10,0$ \\
\hline Glicemia de Jejum e Imur & & & & \\
\hline Glicemia, mg/dL & $96,5 \pm 18,3$ & $90,5 \pm 12,6$ & $89,1 \pm 17,0^{*}$ & $98,4 \pm 15,3$ \\
\hline CV detectável & $56,5 \%$ & $43,5 \%$ & $50 \%$ & $43,5 \%$ \\
\hline Linfócitos CD4, cels $/ \mathrm{mm}^{3}$ & $473,5 \pm 262,8$ & $628,0 \pm 394,0$ & $545,1 \pm 306,9$ & $561,0 \pm 222,0$ \\
\hline Linfócitos CD8, cels $/ \mathrm{mm}^{3}$ & $1002,1 \pm 604,5$ & $1317,0 \pm 691,0$ & $1085,0 \pm 446,1$ & $1175,5 \pm 549,1$ \\
\hline Ingestão alimentar & & & & \\
\hline Carboidratos, g & $235,4 \pm 20,0$ & $225,7 \pm 17,6$ & --- & --- \\
\hline Proteínas, g & $70,3 \pm 5,4$ & $68,2 \pm 8,1$ & --- & --- \\
\hline Lipídios, g & $64,8 \pm 7,8$ & $66,3 \pm 7,7$ & --- & --- \\
\hline Energia, Kcal & $1806,0 \pm 109,4$ & $1772,0 \pm 132,2$ & --- & --- \\
\hline
\end{tabular}

A Tabela 2 apresenta os resultados para variáveis antropométricas, composição corporal e ingestão alimentar para os grupos TR e Controle antes e após a intervenção. As covariantes não evidenciaram ajustes significativos sobre as medidas de pré-testes para a pressão arterial diastólica e glicemia. Os resultados sugerem redução significativa $(P<0,01)$ no perímetro de cintura e massa corporal total, IMC, percentual de 
gordura e massa corporal gorda no grupo TR comparado ao Controle.

Em comparação ao controle, o TR melhorou a força de $1 \mathrm{RM}$, cerca de $49 \%$ no agachamento;
$13 \%$ no supino reto; $34,1 \%$ na cadeira extensora; $51 \%$ no tríceps; $37,2 \%$ na cadeira flexora e $60 \%$ na rosca bíceps (Tabela 3).

Tabela 3. Valores de linha de base e após 24 semanas para as medidas de força muscular $(\mathrm{Kg})$.

\begin{tabular}{lrrrr}
\hline & \multicolumn{2}{c}{ TR $(\mathbf{n}=\mathbf{2 3})$} & \multicolumn{2}{c}{ Controle $(\mathbf{n}=\mathbf{2 2})$} \\
\cline { 2 - 5 } & \multicolumn{1}{c}{ Pré } & Pós & Pré & Pós \\
\hline Agachamento & $21,0 \pm 4,9$ & $31,2 \pm 5,1^{*}$ & $20,0 \pm 4,5$ & $20,4 \pm 4,5$ \\
Supino & $34,3 \pm 8,1$ & $39,8 \pm 9,4^{*}$ & $38,9 \pm 7,2$ & $36,9 \pm 9,9$ \\
Cadeira Extensora & $26,3 \pm 7,1$ & $37,1 \pm 6,6^{*}$ & $26,4 \pm 8,1$ & $27,3 \pm 8,8$ \\
Tríceps & $22,9 \pm 4,0$ & $38,3 \pm 4,9^{*}$ & $23,2 \pm 4,6$ & $23,6 \pm 5,0$ \\
Pulley costas & $31,7 \pm 3,9$ & $41,7 \pm 4,4^{*}$ & $32,3 \pm 8,5$ & $31,4 \pm 9,0$ \\
Mesa Flexora & $18,9 \pm 3,4$ & $27,3 \pm 3,2^{*}$ & $20,0 \pm 5,1$ & $20,2 \pm 4,2$ \\
Rosca Bíceps & $27,9 \pm 6,9$ & $40,4 \pm 4,5^{*}$ & $26,4 \pm 5,9$ & $25,9 \pm 4,4$ \\
\hline Dados apresentados como a média $\left( \pm\right.$ desvio-padrão). ${ }^{*}=$ diferença significativa $P<0,05$.
\end{tabular}

Observou-se significativo $(P<0,05)$ aumento na área muscular do braço isenta de massa óssea, aproximadamente $19 \%$, para o grupo TR e redução, em $8,4 \%$, para o controle. A Figura 1 apresenta a evolução da estimativa de área muscular isenta de massa óssea do braço direito para os grupos.

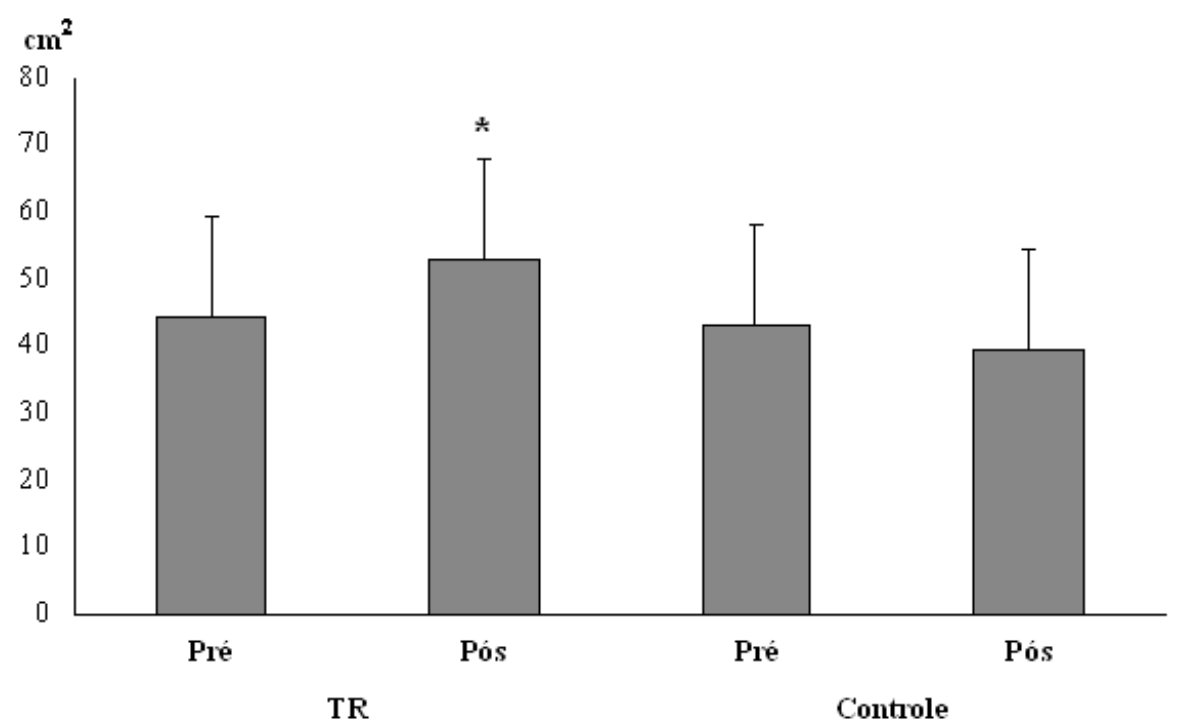

Figura 1. Medidas da área muscular do braço direito isenta de massa óssea $\left(\mathrm{cm}^{2}\right)$.

\section{Discussão}

$\mathrm{Na}$ medida em que progride a infecção pelo HIV, ocorre progressiva redução da massa corporal magra (MACERA, 2007). Neste sentido, torna-se relevante investigar os efeitos crônicos do TR, visando à constituição de massa muscular e ganho de força (SATTLER et al., 2002; SOUZA et al., 2008). O presente estudo recomenda o TR como terapia auxiliar no tratamento da infecção pelo HIV, haja vista que os principais resultados revelaram que 24 semanas aumentaram, significativamente, a força e estimativa de hipertrofia muscular do braço direito. Ademais, observou-se redução significativa da gordura corporal, glicemia de jejum e perímetro de cintura. Estas variáveis estão relacionadas com as manifestações adversas da HAART. Embora outros estudos tenham investigado os efeitos do TR em grupos de HIV soropositivos (LINDEGAARD et al., 2008; SAKKAS et al., 2009; SATTLER et al., 2002; SOUZA et al., 2008), não é de nosso conhecimento trabalhos que realizaram prescrição nutricional onde os mesmos foram submetidos à atividade física. É importante ressaltar que aspectos nutricionais devem ser 
considerados para a verificação dos efeitos do TR sobre a síntese de proteínas e reconstituição do glicogênio muscular e hepático (VIEILLEVOYE et al., 2010), principalmente em pacientes com propensão à degeneração muscular.

Certamente o uso da HAART, por longo período, aumenta a propensão à dislipidemia, resistência à insulina e risco cardiovascular (CAHN et al., 2010). Por outro lado, o TR é citado como intervenção não-medicamentosa para 0 controle da glicemia e redução da resistência à insulina (BLACK et al., 2010). Poehlman et al. (2002) evidenciaram que o TR auxilia no controle glicêmico, uma vez que a glicose é prioritariamente utilizada para a reposição do glicogênio muscular. De fato, nossos resultados corroboram com estudos acima mencionados, pois a glicemia de jejum reduziu cerca de $6,6 \%$ no grupo TR e elevou-se em 10,4\% para o Controle. Em contrapartida, Dolan et al., (2006) não se observaram efeito significativo do treinamento concorrente (30 min. de aeróbico + 6 exercícios, sendo $3 \times 10$ repetições a $70-80 \%$ de $1 \mathrm{RM}$ ) no controle glicêmico de mulheres HIV-soropositivas após 16 semanas. Outro estudo com duração similar, Robinson et al. (2007) também mediram a influência do TR (3 sessões/semana) no controle glicêmico de HIV-soropositivos, porém não foram observadas diferenças significativas após a intervenção. Segundo Dolan et al. (2006), a baixa influência do TR no controle glicêmico, possivelmente está associada ao pequeno tempo de intervenção nos estudos com esta população e a ausência de estimulo aeróbico.

Observou-se aumento significativo da força voluntária máxima em todos os grupos musculares exercitados. Estes resultados são consistentes com os estudos de Lindegaard et al. (2008), Sakkas et al. (2009), Souza et al. (2008), Souza et al. (2011). O aumento da força muscular é extremamente importante para o metabolismo corporal de HIV soropositivos, uma vez que é diretamente associado ao aumento da taxa metabólica basal e do catabolismo de lipídios na região abdominal (ORMSBEE et al., 2007). Melanson et al. (2002) observaram aumento em $5 \%$ na lipólise, após sessão única de exercício resistido. Possivelmente a redução significativa do perímetro de cintura e do percentual de gordura, observados em nosso estudo, sejam decorrentes da lipólise advinda da adaptação ao TR. Após 16 semanas de TR ( 3 sessões/semana), Robinson et al. (2007) verificaram redução significativa na gordura do tronco em HIV-soropositivos submetidos a uso crônico da HAART (>24 meses). Sabe-se que contrações musculares crônicas intermitentes induzem ao aumento dos níveis circulantes de miocinas, em especial a interleucina-6, que promove a lipólise e oxidação de ácidos graxos (PETERSEN; PEDERSEN, 2005).

Em relação ao aumento de força pósintervenção, o menor percentual de ganho foi observado no supino reto. Possivelmente esta resposta esteja associada à constituição da amostra, uma vez que, aproximadamente $70 \%$, eram mulheres. Após doze semenas de TR em mulheres diabéticas, Kwon et al. (2010) observaram aumento médio em $13,2 \%$ na força de membros superiores e $17,2 \%$ nos inferiores. O aumento na força parece estar associado aos padrões geneticamente determinados em homens e mulheres. Liu et al. (2010) observaram que o gênero é fator determinante na regulação transcricional do bíceps braquial, pois o TR resulta em diferentes padrões de hipertrofia e força muscular. Mulheres tendem a apresentar rápida regeneração pós-treino e homens apresentam sinalização hipertrófica mais prolongada. Ademais, mulheres tendem a expressar maior força absoluta nos membros inferiores quando comparada aos superiores (SIMÃO et al., 2007).

A avaliação do perímetro de cintura é relevante, uma vez que reflete o estoque de gordura visceral decorrente da HAART (HAKEEM et al., 2010; DE SAINT MARTIN et al., 2006). Neste sentido, os resultados de nosso estudo sugerem que a redução do perímetro de cintura, observada no TR, pode ter contribuído para menores valores pressóricos em relação ao grupo fisicamente inativo. Scevola et al. (2003) ressaltam a importância do TR para melhora da capacidade cardiovascular em soropositivos submetidos à HAART. Tratando-se do risco cardiometabólico, dados do Framingham Heart Study (PREIS et al., 2010) evidenciaram correlação positiva entre o perímetro de pescoço e a gordura visceral. Em outras palavras, a cada incremento de $1,2 \mathrm{~cm}$ de pescoço, eleva-se o risco cardiovascular em cerca de uma unidade. Embora nosso estudo não tenha revelado diferenças significativas entre os grupos, sobre esta variável dependente verificou-se redução superior a $1 \mathrm{~cm}$ para o TR em relação aos valores de linha de base. 
Além da força, observou-se significativo aumento da área muscular do braço direito isenta de massa óssea. Similar ao presente estudo, Robinson et al. (2007) verificaram aumento em $3,9 \%$ ( 2 kgs) na massa corporal magra, em HIVsoropositivos, submetidos a 16 semanas de treinamento concorrente. A medida por absortometria de raios- $X$ de dupla energia indicou a hipertrofia periférica como principal responsável pelo aumento da massa magra. Em outro estudo com treinamento concorrente, Dolan et al. (2006) observaram $6 \%$ de hipertrofia pós-intervenção (16 semanas). A hipertrofia de membros é de importância em pacientes com lipoatrofia periférica (GUARALDI et al., 2006), uma vez que esta condição contribui para percepção negativa da imagem corporal e eleva a propensão a distúrbios do humor como ansiedade e depressão (COLLINS et al., 2000). Sullivan et al. (2003) observaram que $39 \%$ dos pacientes em tratamento com inibidores de transcriptase reversa apresentam fadiga e depressão. Nota-se que soropositivos tendem a apresentar reduzida síntese proteica e balanço nitrogenado negativo quando comparados a indivíduos saudáveis (ROUBENOFF et al., 2001).

É importante destacar que o protocolo adotado durante a intervenção não resultou em imunossupressão. Estudos de duração inferior (LINDEGAARD et al., 2008) ou similar ao presente trabalho (MUTIMURA et al., 2008) não observaram alterações na imunidade de soropositivos associadas ao exercício. Neste sentido, sugere-se que a modulação da resposta imune está associada ao uso da HAART que, apesar dos efeitos colaterais, mostrou-se eficiente em normalizar os níveis de linfócitos $\mathrm{CD} 4^{+}$e $\mathrm{CD} 8^{+}$ e controlar a carga viral (CICCOLO et al., 2004; DUDGEON et al., 2004). Sendo assim, o TR pode ser utilizado como estratégia de controle para os efeitos adversos do uso crônico da HAART. Lox et al. (1995) observaram que o TR resulta em melhora nos indicadores de qualidade de vida em HIV-soropositivos o que, por sua vez, contribui positivamente para a adesão ao tratamento. Apesar do presente estudo ter utilizado grupos mistos quanto às variáveis gênero e lipodistrofia, acreditamos que amostras heterogêneas são mais representativas. Ademais, a ausência do cálculo amostral limitam os resultados. Outra limitação refere-se à categorização da variável contínua carga viral. Embora este seja um procedimento comum em estudos clínicos, pode refletir negativamente no poder estatístico dos testes - power. Sugere-se, para futuros estudos, a análise da gordura visceral, comparação entre indivíduos com ou sem lipodistrofia e a adoção de outros métodos de treino, dentre eles o concorrente e KAATSU.

\section{Conclusões}

Mediante os resultados do presente estudo, conclui-se que 24 semanas de TR resultaram em melhora na força e hipertrofia. Sem contar que o treinamento aplicado contribuiu para o controle de variáveis metabólicas que são afetadas pelo uso crônico da HAART, uma vez que esta terapia é inevitável ao HIV-soropositivo. O exercício físico deve ser recomendado para dirimir efeitos colaterais, bem como resultar em benefícios à saúde do paciente.

\section{Referências}

ACSM. American College of Sports Medicine position stand. Progression models in resistance training for healthy adults. Medicine and Science in Sports and Exercise, Madison, v.41, n.3, p.364-380, 2009. DOI:

http://dx.doi.org/10.1249/MSS.0b013e3181915670

AGIN, D.; GALLAGHER, D.; WANG, J.; HEYMSFIELD, S.B.; PIERSON, R.N. JR., KOTLER, D.P. Effects of whey protein and resistance exercise on body cell mass, muscle strength, and quality of life in women with HIV. AIDS, London, v.15, n.18, p.2431-2440, 2001. Disponível em:

http://pdfs.journals.Iww.com/aidsonline/2001/1207 0/Effects of whey protein and resistance exerci se on.11.pdf. Acesso em: 23 mar 2013.

BLACK, L.E.; SWAN, P.D., ALVAR, B.A. Effects of intensity and volume on insulin sensitivity during acute bouts of resistance training. Journal of Strength and Conditioning Research, Champaign, v.24, n.4, p.1109-16, 2010. DOI: http://dx.doi.org/10.1519/JSC.0b013e3181cbab6d.

BORG, G. Psychophysical bases of perceived exertion. Medicine and Science in Sports and Exercise, Madison, v.14, n.5, p.377-387, 1982.

CADE, W.T., REEDS, D.N.; MITTENDORFER, B.; PATTERSON, B.W.; POWDERLY, W.G.; KLEIN, S.; YARASHESKI, K.E. Blunted lipolysis and fatty acid oxidation during moderate exercise in HIVinfected subjects taking HAART. American journal of physiology. Endocrinology and metabolism, Bethesda, v.292, n.3, p.E812-E819, 2007. DOI:

http://dx.doi.org/10.1152/ajpendo.00300.2006 
CAHN, P.; LEITE, O.; ROSALES, A.; CABELLO, R.; ALVAREZ, C.A.; SEAS, C.; CARCAMO, C.; CURE-BOLT, N.; L'ITALIEN, G.P.; MANTILLA, P.; DEIBIS, L.; ZALA, C.; SUFFERT, T. Metabolic profile and cardiovascular risk factors among Latin American HIV-infected patients receiving HAART. The Brazilian Journal of Infectious Diseases. Salvador, v.14, n.2, p.158-166, 2010. Disponível em:

http://www.scielo.br/pdf/bjid/v14n2/v14n2a08.pdf. Acesso em: 23 mar 2013.

CICCOLO, J.T.; JOWERS, E.M.; BARTHOLOMEW, J.B. The benefits of exercise training for quality of life in HIV/AIDS in the postHAART era. Sports Medicine, Auckland, v.34, n.8, p.487-499, 2004.

COLLINS, E.; WAGNER, C.; WALMSLEY, S. The AIDS Reader. New York, v.10, n.9, p.546-550, 2000.

COUZIGOU, C.; SEMAILLE, C.; STRAT, Y.L.; PINGET, R.; PILLONEL, J.; LOT, F.; CAZEIN, F.; VITTECOQ, D.; DESENCLOS, J.C. Differential improvement in survival among patients with AIDS after the introduction of HAART. AIDS Care. London, v.19, n.4, p.523-531, 2007. DOI: http://dx.doi.org/10.1080/09540120701235628.

DE SAINT MARTIN, L.; VANDHUICK, O.; GUILLO, P.; BELLEIN, V.; BRESSOLLETTE, L.; ROUDAUT, N.; AMARAL, A.; PASQUIER, E. Premature atherosclerosis in HIV positive patients and cumulated time of exposure to antiretroviral therapy (SHIVA study). Atherosclerosis. Amsterdam, v.185, n.2, p.361-367, 2006. DOI: http://dx.doi.org/10.1016/i.atherosclerosis.2005.06 $\underline{.049 .}$.

DELL'ISOLA, C.; APREA, L.; PIZZELLA, T.; IZZO, C. Effect of anti-retroviral therapy on body composition changes: a literature review. Le Infezioni in Medicina, Roma, v.14, n.1, p.5-12, 2006.

DOLAN, S.E.; FRONTERA, W.; LIBRIZZI, J.; LJUNGQUIST, K.; JUAN, S.; DORMAN, R.; COLE, M.E.; KANTER, J.R.; GRINSPOON, S. Effects of a supervised home-based aerobic and progressive resistance training regimen in women infected with human immunodeficiency virus: a randomized trial. Archives of Internal Medicine, Chigago, v.166, n.11, p.1225-1231, 2006. DOI: http://dx.doi.org/10.1001/archinte.166.11.1225.

DUDGEON, W.D.; PHILLIPS, K.D.; BOPP, C.M.; HAND, G.A. Physiological and psychological effects of exercise interventions in HIV disease. AIDS Patient Care and STDS. Larchmont, v.18, n.2, p.81-98, 2004. DOI: http://dx.doi.org/10.1089/108729104322802515.
DUONG, M.; PETIT, J.M.; MARTHA, B.; GALLAND, F.; PIROTH, L.; WALLDNER, A.; GRAPPIN, M.; BUISSON, M.; DUVILLARD, L.; CHAVANET, P.; PORTIER, H. Concentration of circulating oxidized LDL in HIV-infected patients treated with antiretroviral agents: relation to HIVrelated lipodystrophy. HIV Clinical Trials, St. Louis, v.7, n.2, p.41-47, 2006.

FLORINDO, A.A.; LATORRE, M.D.O.; SANTOS, E.C.; BORELLI, A.; ROCHA, M.D.E.;

SEGURADO, A.A. Validation of equations of skinfold thickness for fat mass estimation in HIV/AIDS subjects: a comparison of dual energy $x$-ray absorptometry and computed tomography of abdomen. Revista Brasileira de Atividade Física e Saúde, Londrina, v.13, n.2, p.75-83, 2008. Disponível em:

http://periodicos.ufpel.edu.br/ojs2/index.php/RBAF S/article/viewFile/786/795. Acesso em: 23 mar 2013.

FRISANCHO, A.R. New standards of weight and body composition by frame size and height for assessment of nutritional status of adults and the elderly. The American Journal Clinical Nutrition. Bethesda, v,40, n.4, p.808-819, 1984.

GUARALDI, G.; ORLANDO, G.; SQUILLACE, N.; DE SANTIS, G.; PEDONE, A.; SPAGGIARI, A.; DE FAZIO, D.; VANDELLI, M.; DE PAOLA, M.; BERTUCELLI, C.; ALDROVANDI, C.; NARDINI, G.; BEGHETTO, B.; BORGHI, V.; BERTOLOTTI, M.; BAGNI, B.; GRAZIA AMORICO, M.; ROVERATO, A.; ESPOSITO, R. Multidisciplinary approach to the treatment of metabolic and morphologic alterations of HIV-related lipodystrophy. HIV Clinical Trials. St. Louis, v.7, n.3, p.97-106, 2006.

HAKEEM, A.; BHATTI'S.; CILINGIROGLU, M. The spectrum of atherosclerotic coronary artery disease in HIV patients. Current Atherosclerosis Report. Philadelphia, v.12, n.2, p.119-124, 2010.

INSTITUTE OF MEDICINE. Dietary Reference Intakes for energy, carboydrate, fiber, fat, fatty acids, cholesterol, protein, and amino acids. Food and Nutrition Board Washington D.C.: National Academic Press, 2005.

INSTITUTE OF MEDICINE. Dietary Reference Intakes for Vitamin C, Vitamin E, Selenium, and Carotenoids. Food and Nutrition Board Washington D.C.: National Academic Press, 2000. Disponível em: http://www.nap.edu/openbook.php?record id=981 ‥ Acesso em: 11 mai 2010.

KOHLER, J.J.; LEWIS, W. A brief overview of mechanisms of mitochondrial toxicity from NRTIs. Environmental and Molecular Mutagenesis, New York, v.48, n.3-4, p.166-172, 2007. 
KRAEMER, W.J.; FRY, A.C. Strength testing: development and evaluation of methodology. In: Maud PJ, Foster C, (eds.). Physiological assessment of human fitness. Champaign: Human Kinetics; 1995. p. 115-138. Disponível em: http://www.humankinetics.com/products/allproducts/physiological-assessment-of-humanfitness-2nd-edition. Acesso em: 11 mai 2010.

LIU, D.; SARTOR, M.A.; NADER, G.A.; GUTMANN, L.; TREUTELAAR, M.K.; PISTILLI, E.E.; IGLAYREGER, H.B.; BURANT, C.F.; HOFFMAN, E.P.; GORDON, P.M. Skeletal muscle gene expression in response to resistance exercise: sex specific regulation. BMC Genomics, London, v.11, n.659, p.1-14, 2010. DOI: http://dx.doi.org/10.1186/1471-2164-11-659

\section{PETROSKI, E. Antropometria: Técnicas e} Padronizações. Porto Alegre: Pallotti, 2009.

KWON, H.R.; HAN, K.A.; KU, Y.H.; AHN, H.J.; KOO, B.K.; KIM, H.C.; MIN, K.W. The effects of resistance training on muscle and body fat mass and muscle strength in type 2 diabetic women.

Korean Diabetes Journal, Seoul, v.34, n.2, p.101-110, 2010. DOI:

http://dx.doi.org/10.4093/kdj.2010.34.2.101.

LICHTENSTEIN, K.A.; WARD, D.J.; MOORMAN, A.C.; DELANEY, K.M.; YOUNG, B.; PALELLA, F.J. JR.; RHODES, P.H.; WOOD, K.C.; HOLMBERG, S.D. Clinical assessment of HIVassociated lipodystrophy in an ambulatory population. AIDS. London, v.15, n.11, p.13891398, 2001. Disponível em:

http://pdfs.journals.Iww.com/aidsonline/2001/0727 0/Clinical assessment of HIV associated.8.pdf. Acesso em: 11 mai 2010.

LINDEGAARD, B.; HANSEN, T.; HVID, T.; VAN HALL, G.; PLOMGAARD, P.; DITLEVSEN, S.; GERSTOFT, J.; PEDERSEN, B.K. The effect of strength and endurance training on insulin sensitivity and fat distribution in human immunodeficiency virus-infected patients with lipodystrophy. The Journal of Clinical and Endocrinology and Metabolism, Springfield, v.93, n.10, p.3860-3869, 2008.

LOX, C.L.; MCAULEY, E.; TUCKER, S. Exercise as an intervention for enhancing subjective wellbeing in an HIV-1 population. Journal of Sport \& Exercise Psychology, Champaign, v.17, n.4, p.345-362, 1995. Disponível em:

http://journals.humankinetics.com/isep-backissues/JSEPVolume17Issue4December/Exercisea sanInterventionforEnhancingSubjectiveWellBeingi nanHIVIPopulation. Acesso em: 11 mai 2010.

MACERA, C.A. A home-based exercise program for women with HIV. Clinical Journal of Sports Medicine New York, v.17, n.2, p.172, 2007.
Disponível em: DOI:

http://dx.doi.org/10.1097/01.jsm.0000277809.9486 $\underline{1 . e 7}$

MATSUDO, S.; ARAÚJO, T.; MATSUDO, V.; ANDRADE, D.; ANDRADE, E.; OLIVEIRA, L.C.; BRAGGION, G. Questionário Internacional de Atividade Física (IPAQ): estudo de validade e reprodutibilidade no Brasil. Revista Brasileira de Atividade Física e Saúde Londrina, v.6, n.2, p.512, 2001.

MELANSON, E.L.; SHARP, T.A.; SEAGLE, H.M.; DONAHOO, W.T.; GRUNWALD, G.K.; PETERS, J.C.; HAMILTON, J.T.; HILL, J.O. Resistance and aerobic exercise have similar effects on 24-h nutrient oxidation. Medicine and Science in Sports and Exercise, Madison, v.34, n.11, p.1793-1800, 2002. DOI:

http://dx.doi.org/10.1249/01.MSS.0000037092.245 $\underline{64.33}$

MENDES, E.L.; RIBEIRO ANDAKI, A.C.; BRITO, C.J.; CÓRDOVA, C.; NATALI, A.J.; SANTOS AMORIM, P.R.; DE OLIVEIRA, L.L.; DE PAULA, S.O.; MUTIMURA, E. Beneficial effects of physical activity in an HIV-infected woman with lipodystrophy: a case report. Journal of Medical Case Reports, London, v.5, n.430, p.1-6, 2011. Disponível em:

http://www.jmedicalcasereports.com/content/pdf/1 752-1947-5-430.pdf. Acesso em: 11 mar 2011.

MUTIMURA, E.; CROWTHER, N.J.; CADE, T.W.; YARASHESKI, K.E.; STEWART, A. Exercise training reduces central adiposity and improves metabolic indices in HAART-treated HIV-positive subjects in Rwanda: a randomized controlled trial. AIDS Research and Human Retroviruses, New, York, v.24, n.1: 15-23, 2008. DOI: http://dx.doi.org/10.1089/aid.2007.0023.

O'BRIEN, K., NIXON, S.; GLAZIER, R.H. ; TYNAN, A.M. Progressive resistive exercise interventions for adults living with HIV/AIDS. Cochrane Database Systematic Reviews, Oxford, v.18, n.4, p.CD004248, 2004.

ORMSBEE, M.J.; THYFAULT, J.P.; JOHNSON, E.A.; KRAUS, R.M.; CHOI, M.D.; HICKNER, R.C. Fat metabolism and acute resistance exercise in trained men. Journal of Applied Physiology. Washington D.C., v.102, n.5, p.1767-1772, 2007.

PETERSEN, A.M.; PEDERSEN, B.K. The antiinflammatory effect of exercise. Journal of Applied Physiology. Washington D.C., v.98, n.4, p.1154-1162, 2005.

PETROSKI, E. Antropometria: Técnicas e Padronizações. Porto Alegre: Pallotti, 2009. 
POEHLMAN, E.T.; DENINO, W.F.; BECKETT, T.; KINAMAN, K.A.; DIONNE, I.J.; DVORAK, R.; ADES, P.A. Effects of endurance and resistance training on total daily energy expenditure in young women: a controlled randomized trial. Journal of Clinical Endocrinology and Metabolism. Springfield, v.87, n.3, p.1004-1009, 2002. DOI: http://dx.doi.org/10.1210/jc.87.3.1004

PREIS, S.R.; MASSARO, J.M.; HOFFMANN, U.; D'AGOSTINO, R.B. S.R.; LEVY, D.; ROBINS, S.J.; MEIGS, J.B.; VASAN, R.S.; O'DONNELL, C.J.; FOX, C.S. Neck Circumference as a Novel Measure of Cardiometabolic Risk: The Framingham Heart Study. Journal of Clinical Endocrinology and Metabolism. Springfield, v.95, n.8, 2010. Disponível em: http://jcem.endojournals.org/cgi/rapidpdf/jc.20091779v1. Acesso em: 23 mar 2013.

ROBINSON, F.P.; QUINN, L.T.; RIMMER, J.H. Effects of high-intensity endurance and resistance exercise on HIV metabolic abnormalities: a pilot study. Biological Research for Nursing, Thousand Oaks v.8, n.3, p.177-185, 2007. DOI: http://dx.doi.org/10.1177/1099800406295520.

RØGE, B.T.; CALBET, J.A.L.; MOLLER, K.; ULLUM, H.; HENDEL, H.W.; GERSTOFT, J.; PEDERSON, B.K. Skeletal muscle mitochondrial function and exercise capacity in HIV-infected patients with lipodystrophy and elevated p-lactate levels. AIDS, London, v. 16, n.7, p.973-982, 2002. Disponível em:

http://pdfs.journals.Iww.com/aidsonline/2002/0503 0/Skeletal muscle mitochondrial function and.3. pdf. Acesso em: 23 mar 2013.

ROUBENOFF, R.; MCDERMOTT, A.; WEISS, L.; SURI, J.; WOOD, M.; BLOCH, R.; GORBACH, S. Short-term progressive resistance training increases strength and lean body mass in adults infected with human immunodeficiency virus.

AIDS, London, v.13, n.2, p.231-239, 1999.

Disponível em:

http://pdfs.journals.Iww.com/aidsonline/1999/0204 0/Short term progressive resistance training.11. pdf. Acesso em: 11 mar 2011.

ROUBENOFF, R.; ABAD, L.W.; LUNDGREN, N. Effect of acquired immune deficiency syndrome wasting on the protein metabolic response to acute exercise. Metabolism, New York, v.50, n.3, p.288-292, 2001.

SAKKAS, G.K.; MULLIGAN, K.; DASILVA, M.; DOYLE, J.W.; KHATAMI, H.; SCHLEICH, T.; KENT-BRAUN, J.A.; SCHAMBELAN, M. Creatine fails to augment the benefits from resistance training in patients with HIV infection: a randomized, double-blind, placebo-controlled study. PLoS One, San Francisco, v.4, n.2, p.e4605, 2009.
SATTLER, F.R.; SCHROEDER, E.T.; DUBE, M.P.; JAQUE, S.V.; MARTINEZ, C.; BLANCHE, P.J.; AZEN, S.; KRAUSS, R.M. Metabolic effects of nandrolone decanoate and resistance training in men with HIV. American Journal of

Physiology. Endocrinology and Metabolism. v.283, n.6, p.E1214-1222, 2002. Disponível em: http://www.medibolics.com/Metabolic\%20effects\% 20of\%20Deca\%20Study.pdf . Acesso em: 23 mar 2013.

Sociedade Brasileira de Cardiologia. V Diretrizes Brasileiras de Hipertensão Arterial. Arquivos

Brasileiros de Cardiologia, São Paulo, v.89, n.3, e24-e79, 2007.

SCEVOLA, D.; DI MATTEO, A.; LANZARINI, P.; UBERTI, F.; SCEVOLA, S.; BERNINI, V.; SPOLADORE, G.; FAGA, A. Effect of exercise and strength training on cardiovascular status in HIV-infected patients receiving highly active antiretroviral therapy. AIDS. London, v.17, Supl.1, p.S123-129, 2003.

SIMÃO, R.; MAIOR, A.S.; NUNES, A.P.L.; MONTEIRO, L.; CHAVES, C.P.G. Variações na Força Muscular de Membros Superior e Inferior nas Diferentes Fases do Ciclo Menstrual. Revista Brasileira de Ciência e Movimento, Brasília, v.15, n.3, p.47-52, 2007.Disponível em: http://portalrevistas.ucb.br/index.php/RBCM/article /viewFile/759/762. Acesso em: 23 mar 2013.

SOUZA, P.M.; JACOB-FILHO, W.; SANTARÉM, J.M.; SILVA, A.R.; LI, H.Y.; BURATTINI, M.N. Progressive resistance training in elderly HIVpositive patients: does it work? Clinics, São Paulo, v.63, n.5, p.619-624, 2008.

SOUZA, P.M.; JACOB-FILHO, W.; SANTARÉM, J.M.; ZOMIGNAN, A.A.; BURATTINI, M.N. Effect of progressive resistance exercise on strength evolution of elderly patients living with HIV compared to healthy controls. Clinics, São Paulo, v.66, n.2, p.261-266, 2011.

http://www.scielo.br/pdf/clin/v66n2/14.pdf. Acesso em: 23 mar 2013.

SULLIVAN, A.K.; BURTON, C.T.; NELSON, M.R.; MOYLE, G.; MANDALIA, S.; GOTCH, F.M.; GAZZARD, B.G.; IMAMI, N. Restoration of human immunodeficiency virus-1-specific responses in patients changing from protease to nonnucleoside reverse transcriptase inhibitor-based antiretroviral therapy. Scandinavian Journal of Immunology, Oslo, v.57, n.6, p.600-607, 2003.

TABACHINICK, B.G.; FIDELL, L.S. Using multivariate statistics. New York:Harper Collins, 2001.

UNAIDS. Joint United Nations Programme on HIV/AIDS (UNAIDS) and World Health 
Organization (WHO): Latin America AIDS

epidemic Updated - Regional Summary 2008.

WHO Library Cataloguing-in-Publication Data:

Genebra, 16p. 2008. Disponível em:

http://data.unaids.org/pub/Report/2008/jc1530 epi

briefs latinamerica en.pdf. Acesso em: 23 mar

2013.

VIEILLEVOYE, S.; POORTMANS, J.R.;

DUCHATEAU, J.; CARPENTIER, A. Effects of a

combined essential amino acids/carbohydrate

supplementation on muscle mass, architecture and maximal strength following heavy-load

training. European Journal of Applied

Physiology, Berlin, v.110, n.3, p. 479-88, 2010.

http://www.springerlink.com/content/4185723k042 22034/fulltext.pdf. Acesso em: 23 mar 2013.

ZIMPEL, R.; FLECK, M.P.A. Quality of life in HIVpositive Brazilians: application and validation of the WHOQOL-HIV, Brazilian version. AIDS Care, London, v.19, n.7, p.923-930, 2007.

Financiamento: CAPES - Coordenação de Aperfeiçoamento de Pessoal de Nível Superior

\section{Endereço:}

Ciro José Brito

Rua Niceu Dantas 1074 casa 07

Aracaju SE Brasil

49037-470

Telefone: (79) 20156536

e-mail: cirojbrito@gmail.com

Recebido em: 10 de agosto de 2010.

Aceito em: 07 de abril de 2013.

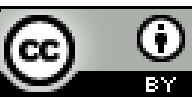

Motriz. Revista de Educação Física. UNESP, Rio Claro,

SP, Brasil - elSSN: 1980-6574 - está licenciada sob

Creative Commons - Atribuição 3.0 